\title{
A Novel liposomal nanoparticle for the imaging of amyloid plaque by MRI
}

\author{
Eric A. Tanifum ${ }^{1}$, Ketan Ghaghada ${ }^{1}$, Craig Vollert ${ }^{2}$, Elizabeth Head ${ }^{3}$, Jason L. Eriksen ${ }^{2}$, and \\ Ananth Annapragada ${ }^{1, *}$ \\ ${ }^{1}$ Texas Children's Hospital, Houston, TX \\ ${ }^{2}$ University of Houston, Houston, TX \\ ${ }^{3}$ University of Kentucky, Lexington, KY
}

\begin{abstract}
Amyloid binding molecules with greater hydrophilicity than existing ligands were synthesized. The lead candidate ET6-21 bound amyloid fibrils, and amyloid deposits in dog brain and human brain tissue ex vivo. The ligand was used to prepare novel amyloid-targeted liposomal nanoparticles. The preparation was tested in the Tg2576 and TetO/APP mouse models of amyloid deposition. Gd chelates and Indocyanine green were included in the particles for visualization by MRI and near-infrared microscopy. Upon intravenous injection, the particles successfully traversed the blood-brain barrier in these mice, and bound to the plaques. Magnetic resonance imaging (T1-MRI) conducted 4 days after injection demonstrated elevated signal in the brains of mice with amyloid plaques present. No signal was observed in amyloid-negative mice, or in amyloid-positive mice injected with an untargeted version of the same agent. The MRI results were confirmed by immunohistochemical and fluorescent microscopic examination of mouse brain sections, showing colocalization of the fluorescent tags and amyloid deposits.
\end{abstract}

\section{Keywords}

Alzheimer's disease; imaging; amyloid plaque; amyloid angiopathy; liposome; magnetic resonance imaging; gadolinium; nanoparticle; molecular imaging

\section{Introduction}

Under the prevailing amyloid cascade hypothesis of Alzheimer's disease (AD), amyloid burden is considered a necessary, but not sufficient, condition for the existence of the

\footnotetext{
*To whom correspondence should be addressed, at: The Singleton Department of Pediatric Radiology, Texas Children's Hospital, 1102 Bates Street, Suite 850, Feigin Center, Houston, TX 77030, Phone: 832-824-0865, avannapr@ texaschildrens.org.

Author Contributions Eric Tanifum: conducted all synthesis, characterization, in vitro and in vivo testing of the particles. Ketan Ghaghada: performed MRI studies, interpreted data. Craig Vollert: conducted histopathological studies on mouse brains. Elizabeth Head: performed ex vivo studies on dog and human brain tissue. Jason Eriksen: oversaw all histopathological studies. Ananth Annapragada: study sponsor and guarantor, oversaw all studies, conducted image data analysis, binding constant evaluation. All authors contributed significantly to manuscript writing, editing and finalization.
}

Competing Financial Interests: ET, JE and AA own stock in Alzeca LLC, a company that has licensed some of the technology described in this paper. 
disease. Methods to detect amyloid plaques in the brain are therefore of interest. Three positron emission tomography (PET) imaging agents that bind specifically to amyloid plaques have recently been approved by the FDA, and can be used for the detection of amyloid plaques. However, their spatial resolution is limited by that of the PET modality, and is on the order of $5-10 \mathrm{~mm}$, limiting any anatomy-specific information available in the image. PET imaging also requires the use of radio-isotopes, and carries the risk of significant radiation: an amyloid PET scan is estimated to expose the patient to about $7 \mathrm{mSv}$ [1] of radiation dose, roughly equivalent to several CT scans (a typical head CT in an adult is about $2 \mathrm{mSv}$ [2]). Global distribution of the PET agents also remains a challenge due to their short half-life. PETNET, the division of Siemens that distributes the majority of PET agents, and handles distribution for Florbetapir (AV-45), shows that 11 entire states in the US, and much of the rest of the world, are not covered in their distribution network [3]. By the last census, this leaves nearly 60 million US residents and even more across the world with very limited access to this technology.

A non-radioactive molecular imaging agent for amyloid plaques, however, is a significant challenge. Assuming intravenous injection the agent would have to cross the blood-brain barrier (BBB), seek out the amyloid plaques, and then generate enough target signal compared to the background noise, to enable accurate detection. Previous efforts to develop an amyloid-targeting MRI agent have primarily focused on either proton T2[4-9] (capitalizing on the high $\mathrm{T} 2$ relaxivities of Iron oxide nanoparticles), or ${ }^{19} \mathrm{~F}$ imaging $[8,10-$ 13], capitalizing on the extremely high signal-to-noise ratios achievable due to the absence of endogenous signal. High T2 relaxivities, however, lead to the suppression of overall signal, making it difficult to reliably quantitate the image (see for example Figure 4b in [8]). ${ }^{19} \mathrm{~F}$ agents have an intrinsic advantage because there is no endogenous, MR-visible fluorine in the body, thus reducing the background signal dramatically, and improving detectability. However, the absence of endogenous MR-visible fluorine also means there is no anatomical landmark in the ${ }^{19} \mathrm{~F}$ image, and a registered ${ }^{1} \mathrm{H}$ MR image is almost always required, to provide anatomical reference [8,14]. We focused in this work, on a Gdcontaining liposomal nanoparticle, with ultra high signal-to-noise ratio, providing sufficient feature conspicuity, while providing an anatomical reference from the background proton signal. Relatively few previous efforts to make a T1 agent for this application have been made $[4,6,15]$, and to our knowledge, none have yet been successful in imaging plaques in vivo following intravenous injection. Kim et al. demonstrated $[10,11,16]$ a hollow manganese oxide nanoparticle targeted using the amyloid peptide as a targeting ligand. It was delivered by intracranial injection into the cisterna magna and did not have to negotiate the blood-brain barrier.

Our previous work $[15,17]$ demonstrated that liposomes containing a derivative of Methoxy$\mathrm{XO} 4$ were capable of penetrating the BBB and binding the majority of amyloid plaques in the APP/PSEN1 mouse, contrary to previous notions of a relatively impenetrable BBB. To determine how these particles were successful in penetrating the BBB, we conducted $[15,16]$ a subsequent study of BBB permeation of untargeted liposomes in the TetO/APP mouse model of AD. This model features the Swedish and Indiana mutations of the APP gene, under the control of a tetracycline responsive element. The mice were maintained on a 
tetracycline-fortified diet for the first 6 months of life, insuring normal maturation of the cerebrovasculature, at which point deletion of the tetracycline from the diet resulted in amyloid deposition. We determined that liposomes penetrated the BBB in both APP+ and APP- mice, with younger $(<9$ mo $)$ APP- mice showing the least BBB penetration, and older APP + mice ( $>18$ months) showing the greatest. Leaks in the choroid plexus and particularly along the dorsomedial cerebellar artery dominated the leak in younger mice independent of amyloid status, while older APP+ mice showed significant increases in leaks in the cerebral cortex. Quantitation of the leaks showed that about a tenth of a percent of payload remained in the mouse brain, even though the payload had no binding ligand to retain it in the brain. This suggested that even larger amounts must have been transported past the BBB in these models. Collectively, these studies therefore suggested that liposomes were successful in penetrating the $\mathrm{BBB}$ of $\mathrm{AD}$ mice, and explained the successful labeling of amyloid plaques by targeted liposomes, further suggesting that detection of amyloid plaques by such targeted liposomal imaging agents is only a matter of sufficient signal-to-noise in the detection. In previous work $[17,18]^{13}$ we demonstrated a liposome with Gd chelates presented on the surface of lipid bilayer, and additional Gd in its interior, with ultra-high per-particle relaxivity on the order of $200,000 \mathrm{mM}^{-1} \mathrm{~s}^{-1}$, at low (1-3T) field strengths. We use this DualGd (core-encapsulated Gd and surface-conjugated Gd) liposomal nanoparticle as the readout mechanism for an MRI amyloid imaging agent.

Existing amyloid binding ligands, including methoxy-XO4 (XO4) are hydrophobic and can interfere with the lipid bilayer in liposomal formulations. When loaded with $\mathrm{Gd}$ chelates for MRI T1 contrast, XO4 targeted liposomes were unstable to the osmotic gradient created by the high Gd chelate internal concentration, and were destabilized. We therefore synthesized a novel series of Methoxy-XO4 analogues, with reduced hydrophobicity. The lead candidate ligand that emerged (ET6-21) enabled stable encapsulation of the Gd chelate within the liposome core and retained the hyper-relaxive surface $\mathrm{Gd}$ molecules in the lipid bilayer.

We tested the ability of ET6-21 conjugated particles to detect amyloid pathology in TetO/APP and Tg2576 mouse models of amyloid deposition. Imaging was performed on a 1.0 T permanent magnet based small animal MRI instrument. Consistent with the histopathological presence of amyloid, signal enhancement in the brains of amyloid-positive mice was observed, whereas this signal was absent in amyloid-negative mice. The binding of the particles to the amyloid pathology was confirmed by colocalization studies using confocal microscopy, following previous work $[15,17]$.

\section{Materials and Methods}

\section{Chemical synthesis}

General-All reagents were obtained from Sigma-Aldrich and used without further purification. Proton nuclear magnetic resonances $\left({ }^{1} \mathrm{H}\right.$ NMR) spectra were recorded at 600 $\mathrm{MHz}$ on a Bruker 600 NMR spectrometer. Carbon nuclear magnetic resonances $\left({ }^{13} \mathrm{C} \mathrm{NMR}\right)$ spectra were recorded at $150 \mathrm{MHz}$ on a Bruker $600 \mathrm{NMR}$ spectrometer. Chemical shifts are reported in parts per million $(\mathrm{ppm})$ from an internal standard acetone $(2.05 \mathrm{ppm})$, chloroform (7.26 ppm), or dimethylsulfoxide ( $2.50 \mathrm{ppm}$ ) for ${ }^{1} \mathrm{H}$ NMR; and from an internal standard of either residual acetone (206.26 ppm), chloroform (77.00 ppm), or 
dimethylsulfoxide (39.52 ppm) for ${ }^{13} \mathrm{C}$ NMR. NMR peak multiplicities are denoted as follows: s (singlet), d (doublet), t (triplet), q (quartet), bs (broad singlet), dd (doublet of doublet), tt (triplet of triplet), ddd (doublet of doublet of doublet), and m (multiplet). Coupling constants $(\mathrm{J})$ are given in hertz $(\mathrm{Hz})$. High resolution mass (HRMS) and MALDI spectra were obtained from Mass Spectrometry Unit of the BioScience Research Collaborative at Rice University, Houston, Texas. Thin layer chromatography (TLC) was performed on silica gel 60 F254 plates from EMD Chemical Inc. and components were visualized by ultraviolet light $(254 \mathrm{~nm})$ and/or phosphomolybdic acid, $20 \mathrm{wt} \%$ solution in ethanol. SiliFlash silica gel (230-400 mesh) was used for all column chromatography.

Synthesis of arylvinylpyrimidines and arylvinylbenzothiazole-A mixture 4Methylpyrimidine $(112 \mu \mathrm{L}, 1.23 \mathrm{mmol})$ or 2-methylbenzothiazole $(156 \mu \mathrm{L}, 1.23 \mathrm{mmol})$, the corresponding aromatic aldehyde $(1.12 \mathrm{mmol})$ and potassium tert-butoxide $(376 \mathrm{mg}, 3.35$ mmol) in $10 \mathrm{~mL}$ DMF were heated at $80{ }^{\circ} \mathrm{C}$ for 12 hours. It was then cooled to ambient temperature and filtered through a pad of celite to remove solids. The resulting filtrate was concentrated and purified by column chromatography on silica gel eluted with ethyl acetate/ hexanes gradient to obtain the desired product.

(E)-2-\{methyl[4-(2-(pyrimidin-4-yl)vinyl)phenyl]amino\}ethanol (ET6-17): 1H NMR $(\mathrm{CDCl} 3,600 \mathrm{MHz}) \delta 8.91(\mathrm{~d}, \mathrm{~J}=1.2 \mathrm{~Hz}, 1 \mathrm{H}), 8.45(\mathrm{~d}, \mathrm{~J}=5.4 \mathrm{~Hz}, 1 \mathrm{H}), 7.70(\mathrm{~d}, \mathrm{~J}=15.6 \mathrm{~Hz}$, $1 \mathrm{H}), 7.41(\mathrm{~d}, \mathrm{~J}=6.6 \mathrm{~Hz}, 2 \mathrm{H}), 7.26(\mathrm{dd}, \mathrm{J}=6.6,1.2 \mathrm{~Hz}, 1 \mathrm{H}), 6.75(\mathrm{~d}, \mathrm{~J}=15.6 \mathrm{~Hz}, 1 \mathrm{H}), 6.67$ (d, $\mathrm{J}=6.6 \mathrm{~Hz}, 2 \mathrm{H}), 3.69(\mathrm{t}, \mathrm{J}=6.6 \mathrm{~Hz}, 2 \mathrm{H}), 3.46(\mathrm{t}, \mathrm{J}=6.6 \mathrm{~Hz}, 2 \mathrm{H}), 3.98(\mathrm{~s}, 3 \mathrm{H}) ; 13 \mathrm{C} \mathrm{NMR}$ (DMSO-d6, 150 MHz) $\delta 163.80,157.60,155.80,150.59,139.19,129.54,123.21,119.48$, $117.83,115.13,111.91,59.13,54.35,38.82$; HRMS clcd for C15H17N3O+m/z (M+H) +256.1444 , found 256.1372 .

(E)-2,2'-\{[4-(2-(pyrimidin-4-yl)vinyl)phenyl]azanediyl\}diethanol (ET6-21): $1 \mathrm{H}$ NMR (MeOD, $600 \mathrm{MHz}) \delta 9.02(\mathrm{~d}, \mathrm{~J}=1.2 \mathrm{~Hz}, 1 \mathrm{H}), 8.61(\mathrm{~d}, \mathrm{~J}=5.4 \mathrm{~Hz}, 1 \mathrm{H}), 7.88(\mathrm{~d}, \mathrm{~J}=15.6 \mathrm{~Hz}$, $1 \mathrm{H}), 7.58(\mathrm{~d}, \mathrm{~J}=8.7 \mathrm{~Hz}, 2 \mathrm{H}), 7.53(\mathrm{dd}, \mathrm{J}=6.0,1.2 \mathrm{~Hz}, 1 \mathrm{H}), 6.98(\mathrm{~d}, \mathrm{~J}=15.6 \mathrm{~Hz}, 1 \mathrm{H}), 6.88(\mathrm{~d}$, $\mathrm{J}=8.7 \mathrm{~Hz}, 2 \mathrm{H}$ ), $3.71(\mathrm{t}, \mathrm{J}=6.0 \mathrm{~Hz}, 4 \mathrm{H}), 3.57$ (t, J=6.0 Hz, 4H); 13C NMR (DMSO-d6, 150 MHz) $\delta 165.23,159.17,157.76,149.72,139.99,130.84,125.87,121.64,119.42,113.66$, 51.36, 50.08; HRMS clcd for C16H19N3O2+m/z (M+H)+ 286.1550, found 286.1546.

(E)-2-methoxy-4-[2-(pyrimidin-4-yl)vinyl]phenol (ET6-47): 1H NMR (DMSO-d6, 600 MHz) $\delta 9.51(\mathrm{bs}, \mathrm{OH}), 9.08(\mathrm{~d}, \mathrm{~J}=1.2 \mathrm{~Hz}, 1 \mathrm{H}), 8.70(\mathrm{~d}, \mathrm{~J}=5.4 \mathrm{~Hz}, 1 \mathrm{H}), 7.86(\mathrm{~d}, \mathrm{~J}=16.2 \mathrm{~Hz}$, $1 \mathrm{H}), 7.54(\mathrm{dd}, \mathrm{J}=5.4,1.2 \mathrm{~Hz}, 1 \mathrm{H}), 7.35(\mathrm{~d}, \mathrm{~J}=1.5, \mathrm{~Hz}, 1 \mathrm{H}), 7.15(\mathrm{dd}, \mathrm{J}=8.0,1.5 \mathrm{~Hz}, 1 \mathrm{H})$, $7.12(\mathrm{~d}, \mathrm{~J}=16.2 \mathrm{~Hz}, 1 \mathrm{H}), 6.83(\mathrm{~d}, \mathrm{~J}=8.0 \mathrm{~Hz}, 1 \mathrm{H}$ ), 3.89 (s, 3H); 13C NMR (DMSO-d6, 150 MHz) $\delta 162.16,158.38,157.39,148.46,147.93,137.25,126.98,122.53,122.21,118.48$, 115.60, 110.71, 55.62; HRMS clcd for C13H12N2O2+m/z (M+H)+ 229.0972, found 229.0981

DSPE-PEG34k-alkyne (4): (See Supplemental Figure S2): To a solution of 1,2distearoyl-sn-glycero-3-phosphoethanolamine-N-[amino(polyethylene glycol)-3400] (DSPE-PEG3400-Amine) $(1.00 \mathrm{~g}, 0.241 \mathrm{mmol})$ in $10 \mathrm{~mL}$ pyridine/chloroform mixture (5 $\mathrm{mL})$, propargylchloroformate $(50 \mu \mathrm{L}, .482 \mathrm{mmol})$ was added and the resulting mixture allowed to stir at ambient temperature overnight. The chloroform was removed in vacuo and 
the resulting residue diluted with $20 \mathrm{~mL} 20 \% \mathrm{EtOH} / \mathrm{H}_{2} 0$ mixture, charged in a $2000 \mathrm{MWCO}$ dialysis bag and dialyzed against MES buffer ( $50 \mathrm{mM}, 5$ litters) for 12 hours and then 2 times against water (5 liters) for 12 hours each. The resulting solution was freeze dried to obtain $1.08 \mathrm{~g}$ of the product as a grey powder, the molecular weight of which was confirmed by MALDI.

DSPE-PEG-3400-Ligand Conjugate (5): (See Supplemental Figure S2): To a solution of alkyne $4(600 \mathrm{mg}, 0.136 \mathrm{mmol})$ and azide $2(84 \mathrm{mg}, 0.273 \mathrm{mmol})$ in $\mathrm{MeOH}(10 \mathrm{~mL})$, THF ( $3 \mathrm{~mL})$ and water $(2 \mathrm{~mL})$ were added, followed by sodium ascorbate $(27.0 \mathrm{mg}, 0.136$ $\mathrm{mmol}$ ) and copper(II)acetate $(2.7 \mathrm{mg}, 0.014 \mathrm{mmol})$. The resulting mixture was stirred at room temperature overnight. The organic solvents were removed invacuo and the resulting residue diluted with $20 \% \mathrm{EtOH} / \mathrm{H} 2 \mathrm{O}$ mixture $(20 \mathrm{~mL})$. This was then loaded in a 2000 MWCO dialysis bag and dialyzed against MES buffer ( $50 \mathrm{mM}, 5$ litters) and then water ( $2 \mathrm{X}$ 5 liters) for 12 hours each. The water was then removed by freeze drying to obtain the product as a white powder $(565 \mathrm{mg})$.

Preparation of liposomes-1,2-dihexadecanoyl-sn-glycero-3-phosphocholine (DPPC) and Cholesterol were purchased from Lipoid Inc., Newark NJ, USA. DSPEPEG3400-ET6-21 was prepared as described above. 1,2-distearoyl-sn-glycero-3phosphoethanolamine-N-[methoxy(polyethylene glycol)-2000] (DSPE-MPEG2000) was purchased from Corden Pharma, Liestahl, Switzerland. DPPC, Cholesterol, DSPEMPEG2000, Bis stearylamine-DTPA-Gd, DSPE-PEG3400-ET6-21 at molar proportions DPPC:BSA-DTPA-Gd: Cholesterol: DSPE-MPEG2000: DSPE-PEG3400-ET6-21 :: 31:25:40:2:2) were dissolved in ethanol to achieve a total concentration of $150 \mathrm{mM}$. A 65 $\mu \mathrm{M}$ solution of ICG was made in $500 \mathrm{mM}$ of gadobenate dimeglumine. The ethanolic solution of lipids was hydrated with a ten-fold excess of the ICG/gadobenate dimeglumine solution at $65^{\circ} \mathrm{C}$ for 30 minutes, allowing multilamellar liposomes to form. The mixture was then extruded in a $10 \mathrm{ml}$ Lipex extruder (Northern Lipids Inc., Burnaby, Canada) using a 200 $\mathrm{nm}$ polycarbonate track-etch filter (10 passes) followed by a $100 \mathrm{~nm}$ polycarbonate filter (10 passes). The suspension was then diafiltered using a MicroKros cross-flow diafiltration cartridge (500kD cutoff), exchanging the external buffer for phosphate buffered saline (PBS, $\mathrm{pH}$ 7.2) for 15 volume exchanges.

Phosphorus and Gd content of the diafiltered product were measured using Inductively Coupled Plasma-Atomic Emission Spectrometry (ICP-AES)-Of the starting Gd, about $20 \%$ remained associated with the liposomes. ICG content was measured by near-IR fluorescence (Ex $780 \mathrm{~nm} / \mathrm{Em} 820 \mathrm{~nm})$. Of the starting ICG, $95 \%$ was estimated to remain associated with the liposomes. ICG content of the diafiltrate was measured at intervals corresponding to complete volume exchanges. The vast majority of the ICG lost from the retentate was present in the first volume exchange, and only trace amounts thereafter.

Dynamic Light Scattering-To measure particle size, periodic samples from the extrusion process, and a final sample after diafiltration, were diluted in PBS and measured on a BI-90 goniometer based DLS system attached to a BI-9000 autocorrelation system. A 
$532 \mathrm{~nm}$ solid state laser was used as the light source, and the concentration of the sample adjusted until discriminated detection with a Hamamatsu photomultiplier tube at $90^{\circ}$ yielded $\sim 100$ kcounts per second (kcps). Correlation functions were measured using an exponentially spaced set of correlator bins, insuring at least 10 channels capturing the initial exponential drop of the correlation function, and 10 channels capturing the long term decay. Correlation functions were averaged for 2 minutes for each sample, using a dustdiscrimination algorithm that eliminated correlation function slices that showed long term correlation functions significantly higher than baseline, indicating contamination with large particle sizes characteristic of dust. The resulting averaged correlation functions were analyzed using the CONTIN algorithm, and the volume averaged distributions used to estimate a mean size and standard deviation. All distributions were practically unimodal $(>=$ $99 \%$ of volume in the main peak).

Stability of the preparations was measured in PBS and reconstituted bovine plasma (RBP)_An aliquot of the preparation was diluted 10x with either PBS or RBP and $200 \mu \mathrm{L}$ of the diluted material placed in a dialysis bag, which was in turn placed in $200 \mathrm{ml}$ PBS and slowly stirred for 24 hours. Samples of the external buffer were taken periodically during the 24 hour period and assayed for Gd content by ICP-AES. Leakage in bovine plasma was $<5 \%$ in both buffer and bovine plasma.

Fibril binding assay-Beta-Amyloid ${ }_{(1-40)}$ peptide was purchased from rPeptide (Bogart, GA), dissolved in PBS at pH 7.4 to a final concentration of $433 \mu \mathrm{g} / \mathrm{ml}(100 \mu \mathrm{M})$. The solution was stirred using a magnetic stir bar at $700 \mathrm{rpm}$ for 4 days at room temperature to drive the formation of fibrils. The stock solution was aliquoted and stored at $-80{ }^{\circ} \mathrm{C}$. The stock solutions were stirred thoroughly prior to removing aliquots for binding assays, to insure a homogenous suspension of fibrils. Aliquots of ET6-21 or ET6-17 solution, or liposomes bearing ET3-73 (and Chrysamine-G for competition assays) were added to achieve the target concentrations for the binding assay, and incubated for 1 hour at room temperature. The incubation mixtures were centrifuged for 20 minutes at 16,400 RPM to separate the fibrils. The precipitate was washed twice with Tris- $\mathrm{HCl}$ and resuspended. Fluorescence was measured using an excitation wavelength of $405 \mathrm{~nm}$ and emission wavelength of $535 \mathrm{~nm}$, to quantify the bound ligands.

Ex vivo tissue staining-ET6-21 solutions in PBS were incubated with frontal cortex tissue obtained from autopsy of 6 confirmed AD patients, with ages at death ranging from 64 to 91 years, and 6 beagle dogs whose ages at death ranged from 12 to 16 years. Both the human and canine tissues had Alzheimer's amyloid pathology confirmed from previous studies. $50 \mu \mathrm{m}$ thick free-floating sections were mounted on slides and allowed to dry overnight. Slides were washed in PBS and incubated in $1 \mathrm{mM}$ ET6-21 solution for 2 hours and then again washed in PBS, and coverslipped in Vectashield mounting medium with DAPI (Vector Laboratories, Burlingame, CA). Sections were imaged on an Olympus BX-51 epifluorescence microscope. The University of Kentucky Institutional Review Board approved the use of human tissue for the study. Informed consent was granted prior to autopsy. 
Relaxometry-Samples with Gd concentrations from 0.25 to $1.0 \mathrm{mM}$ were prepared by diluting liposomal preparations in PBS. T1 relaxation measurements were then performed on a $60 \mathrm{~Hz}$ Bruker minispec MQ benchtop relaxometer (Bruker Instruments, Billerica, MA). Longitudinal relaxation times (T1) were obtained using an inversion recovery sequence. A plot of relaxation rate (1/T1) against Gd concentration yielded a straight line with slope defined as the T1 relaxivity (r1). For relaxivity measurements at higher field strengths (1.5T to $9.4 \mathrm{~T}$ ), the same samples were imaged on corresponding MRI instruments using T1weighted fast spoiled gradient echo (FSPGR) sequence. The slope of the 1/T1 vs. concentration plot was used to estimate the relaxivity at each field strength.

Magnetic Resonance Imaging-The test population consisted of a total of 8 animals with amyloid pathology, and 12 animals without amyloid pathology. Tg2576 mice were 910 month old retired breeders purchased from Taconic Inc., and included 7 APP+ and 5 APP - animals. Of the APP+ animals, 2 were positive by histopathology for amyloid, while 5 were negative. TetO/APP mice consisted of $6 \mathrm{APP}+$ and $2 \mathrm{APP}-$ animals, 12-15 months old; all $6 \mathrm{APP}+$ animals were positive by histopathology for amyloid while both negative animals showed no amyloid pathology. All APP- animals were age-matched non-transgenic littermates of APP+ animals in this study. TetO/APP mice were a kind gift of Dr. Joanna Jankowsky at Baylor College of Medicine. They feature the Swe and Ind mutations of APP, under the control of a Tetracycline promoter. Maintaining them on a Doxycycline enriched diet till the age of 6 weeks allowed normal maturation without amyloid deposition, following which a switch to a normal diet allowed amyloid deposition to occur till the time of imaging. All animal experiments conducted at the Baylor College of Medicine were approved by the Baylor Institutional Animal Care and Use Committee. All animal experiments at MD Anderson Cancer Center were approved by the MDA Institutional Animal Care and Use Committee. All studies were in conformance with the NC3RSARRIVE criteria. All animals studied are reported on in this paper, no animals were excluded from the study.

Tg2576 mice were imaged on a 1.0T Bruker Icon system (Bruker Instruments, Billerica, $\mathrm{MA})$ at MD Anderson Cancer Center. All TetO/APP mice were imaged on an 1.0T Aspect M2 system (Aspect Imaging, Shoham, Israel). Note that these two scanner instruments are built on identical hardware, both manufactured by Aspect Imaging. Only the software interface is different. All imaging parameters were identical on the two instruments. Anesthesia was induced using 5\% isoflurane. Mice were then placed on a custom fabricated sled with integral face-cone for continued anesthesia delivery by inhalation (1.5-3\% isoflurane in air) and transferred to the magnet cavity. A pre-contrast image of the brain was acquired using a 2D multi-slice spin echo sequence $(\mathrm{TE}=32 \mathrm{~ms}, \mathrm{TR}=770 \mathrm{~ms}$, Slice Thickness $=1.2 \mathrm{~mm}, \mathrm{FOV}=30 \times 30 \mathrm{~mm}$, matrix $=156 \times 156,24$ slices A-P, NEX=2). Mice were allowed to awaken and returned to their cages. The mice were injected with contrast liposomes 24 hours after the pre-contrast image. All 8 animals with amyloid pathology, and 9 of the 12 animals with no amyloid pathology received ET6-21 targeted liposomes (150 $\mathrm{mM}$ total lipid concentration, $37.5 \mathrm{mM}$ total Gd concentration, injection volume $4 \mu \mathrm{L} / \mathrm{g}$ body weight, $0.15 \mathrm{mmol} \mathrm{Gd} / \mathrm{kg}$ ). The 3 remaining amyloid negative animals received and equal volume of untargeted (i.e. no ET6-21 present) liposomes with otherwise identical 
composition. 4 days after injection, the mice were anesthetized and imaged again using an identical sequence. The pharmacokinetics of Stealth liposomes in mice are well known [17,19], and a t1/2 of $\sim 24$ hours is widely accepted. A 4-day interval was therefore chosen to insure clearance of the unbound agent from the bloodstream, thus maximizing the contrastto-noise ratio.

Histology-Immediately after the 4-day post images were acquired, the mice were sacrificially perfused with saline, followed by $10 \%$ formalin, the brain excised and submerged in $10 \%$ formalin for 24 hours, then cryoprotected in $30 \%$ sucrose. Whole brain NIR imaging was performed at this stage. Following imaging, the brain was embedded in OCT and cut at $30 \mu \mathrm{m}$ thickness. For amyloid antibody staining, sections were incubated in $5 \%$ normal donkey serum for 1 hour, followed by incubation with anti-amyloid $\beta$ antibody (4G8, from Sigma-Aldrich, St. Louis, MO) in $3 \%$ NDS at $4{ }^{\circ} \mathrm{C}$ overnight. Sections were then washed, and incubated with a FITC or Cy5-tagged dylight649 anti-mouse IgG secondary antibody, mounted and coverslipped using Vectashield mounting medium (Vector Laboratories, Burlingame $\mathrm{CA}$ ) and imaged using the appropriate filter set.

\section{Results}

\section{Novel hydrophilic ligands to amyloid plaque}

Table 1 shows the cLogP values for known amyloid binding molecules XO4, SB-13, and AV-45 in comparison to our new ligands. While Methoxy-XO4 exhibits high affinity for amyloid plaques, we observed from reducing the triaryl- structure to stilbene derivatives such as SB-13 and the clinically used $\mathbf{A V - 4 5}$ results in a corresponding reduction of the clogP value by over 1.5 , yet these molecules maintain binding affinity and specifity to amyloid aggregates. We reasoned that replacing the o-pyridyl ring in $\mathbf{A V - 4 5}$ with bezothiazyl, or pyrimidyl moieties may not only generate analogues with lower clogP, but also ones with increased $\mathrm{H}$-bonding potential. In addition, capping the nitrogen on ring 2 with hydroxyethyl group(s) could further increase the hydrophilicity and increase the potential for the ligand to remain floating in an aqueous medium instead of flipping into the lipid bilayer. We therefore synthesized the new ligands shown in Table 1 in a one step reaction (Supplemental Figure S1), ET6-17, ET6-21, and ET6-47 had the three lowest cLogP values (1.80, 1.06 and 1.56 respectively) and spectrofluorometric testing showed that ET6-17 and ET6-21 both had absorption and emission maxima at $404 \mathrm{~nm}$ and $550 \mathrm{~nm}$, while ET6-47 exhibited an absorption maximum at $346 \mathrm{~nm}$, but no visible emission maximum. We therefore focused on ET6-17 and ET6-21 for further testing because of ease of detection by fluorescence in our in vitro assays and low cLogP values.

Both ET6-17 and ET6-21 bind stably to amyloid fibrils in suspension, and are competed away by Chrysamine-G as shown in Figure 1A and 1B. Consistent with their structural similarity to AV-45 and SB-13, this suggests that they are indeed capable of binding to amyloid plaques, in spite of their relative hydrophilicity. ET6-21 was chosen for subsequent testing due to its superior hydrophilicity. The ET6-21 moiety was then conjugated to a DSPE-PEG3400 anchor using click chemistry, as shown in Supplemental figure S1, yielding ET3-73, the DSPE-PEG conjugate that can be stably maintained in the bilayer of a 
liposome. The binding constants for ET6-17, ET6-21, and liposomes bearing ET3-73 are calculated from the binding curves (Supplemental figure S3). Regressed values are as follows: ET6-17 $\mathrm{Kd}=5.0 \mu \mathrm{M}$, ET6-21 $\mathrm{Kd}=5.0 \mu \mathrm{M}$, ET3-73 $\mathrm{Kd}=3.3 \mu \mathrm{M}$.

The ability of ET6-21 to bind amyloid pathology was tested using human brain tissue obtained from autopsies of 6 human AD patients, whose ages at death ranged from 64 to 92 years. All 6 tissue samples showed clear staining by ET6-21 in a focal pattern consistent with the staining of amyloid plaques. An example is shown in Figure 1C. ET6-21 was also tested in brain tissue samples from 6 beagle dogs with ages at death ranging from 12 to 16 years. Aged canines are a natural model of spontaneous beta-amyloid deposition [16,18]. 4 of 6 samples showed clear labeling of amyloid plaques and CAA. An example is shown in Figure 1D.

\section{Hydrophilic ligands facilitate the formation of stable liposomes}

Table 2 shows the mean diameters and polydispersity indices of liposomes prepared with the XO4 targeting ligand, the ET6-21 ligand, and with no ligand (i.e. PEGylated liposomes), all with bis-stearylamide-DTPA-Gd in the bilayer, and Gd-BOPTA (Gadobenate dimeglumine) in the liposome core. The remainder of the liposome matrix consisted of DPPC (55\%) and Cholesterol (40\%). When extruded through $100 \mathrm{~nm}$ track-etch membranes, a mean diameter around $100 \mathrm{~nm}$ and a low polydispersity index are expected, as exhibited by the PEGylated liposomes. The XO4 liposomes however, exhibit a larger diameter, consistent with interference of the $\mathbf{X O 4}$ ligand with the bilayer, confirmed by negative stain electron microscopy (see supplemental figure S2). The replacement of the XO4 ligand with ET6-21 (Supplemental Figure S1) resulted in liposomes that were once again close to $100 \mathrm{~nm}$ in diameter, with a low polydispersity. On incubation with reconstituted bovine plasma at $37^{\circ} \mathrm{C}$ for 24 hours (plasma leak test), the Gd remained practically $100 \%$ associated with the particles, confirming that the modified ligand yielded a stable bilayer.

\section{Dual-Gd liposomes exhibit extremely high relaxivity}

Liposomes presenting surface $\mathrm{Gd}$ chelates ( $\mathrm{Gd}$ chelates conjugated to a lipid anchor bisstearoyl amine-DTPA-Gd, the bis-stearoyl chains insert into the bilayer) demonstrate high Gd molar relaxivity at low field strengths as shown in Figure 2, consistent with our previous work $[15,17]$. At higher field strengths however, this enhancement of relaxivity is reduced, and at the highest field strength tested (9.4T) the surface Gd is less relaxive than the free chelate. Taking advantage of the relaxivity enhancement at low field strengths, we tested formulations with up to $25 \%$ of the liposome bilayer consisting of BSA-DTPA-Gd. The liposomes were stable, exhibiting no measurable loss of Gd upon storage under refrigerated conditions for up to 8 weeks. The resulting particles have extremely high relaxivity on a perparticle basis, as shown in Figure 2. On a particle basis, a relaxivity of $\sim 190,000 \mathrm{mM}^{-1} \mathrm{~s}^{-1}$ is achieved at $1 \mathrm{~T}$.

\section{ET3-73 targeted dual-Gd liposomes target amyloid plaque and are visible by T1MR in 2 mouse models}

Dual-Gd liposomes, targeted to amyloid plaques using the ET3-73 conjugate were therefore tested for their ability to image amyloid plaques in mice. When injected intravenously into 
mice, and scanned 4-days later using a T1-weighted spin echo sequence ( $T E=32 \mathrm{~ms}$, $T R=770 \mathrm{~ms}$ ) on a 1T MRI scanner, these particles resulted in signal enhancement in patterns characteristic of amyloid deposition, in two distinct strains of amyloid bearing mice (15 month old TetO/APPswe-ind and 9 month old Tg2576). Pre-injection scans of the same mice and amyloid-negative mice similarly injected, as well as amyloid-positive mice injected with untargeted particles, yielded no signal in comparison. The 4-day delay is designed to allow unbound liposomes to clear from the circulation. PEGylated liposomes have a circulation $\mathrm{t} 1 / 2$ of about 24 hours, and our past experience suggested this delay was sufficient for near complete elimination from the blood pool [16,20]. Example images from this study are shown in Figure 3. 6/6 animals in the TetO/APPswe-ind group were consistent with these images as in Figure 3a and b. 2/6 Tg2576 APP+ animals, were also consistent, showing characteristic enhancement as in Figure $3 \mathrm{c}$ and d. The remaining 4/6 showed no enhancement, had no detectable amyloid by 4G8 immunohistochemistry, and were indistinguishable from the non-transgenic sibling animals. There was therefore a $100 \%$ concordance between the presence of amyloid deposits and positive MRI signal, as summarized in Table 3. Overall, following injection of the ET3-73 targeted liposomes, 8/8 animals with amyloid pathology also showed MR signal enhancement, 9/9 with no amyloid pathology showed no MR signal enhancement, and 3/3 injected with untargeted liposomes showed no signal enhancement either.

The liposomes included $\sim 0.6$ mole\% Indocyanine Green (ICG) in the bi-layer for nearinfrared imaging. Figure 4 shows results from near infrared imaging of mouse brains post mortem. Confocal imaging of brain sections from Tg2576 mice revealed enhanced ICG signal in the cortex and hippocampus of the transgenic mice when compared to the nontransgenic controls (A,B), and at high magnification revealed the characteristic punctate structure of amyloid deposits (E,F). The whole brains of TetO/APP+ mice showed greater near infrared fluorescence than their APP- siblings (C,D). Using a fluorescently labeled 4G8 antibody to amyloid plaque, colocalization of the antibody (green), ICG (red) and ET3-73 (blue) is demonstrated for a cluster of 3 plaques (G1-G4) and for an individual plaque (H1-H4). As in the case of the MRI signal, the fluorescence signal was also consistent with the immunohistochemical measure of amyloid pathology presence, as summarized in Table 3. Using Cohen's Kappa to qualify the correlation between immunohistochemistry and MRI signal, p:H0 testing the null hypothesis that the two modalities are independent yields $\mathrm{p}=3.87 \times 10^{-6}$, strongly supporting the equivalence of the MRI and immunohistochemistry measurements. These results confirm that ET3-73 targeted Dual-Gd liposomes can penetrate the BBB, bind the amyloid plaques and generate sufficient signal enhancement for detection using MRI at clinical field strength. The sample size at which a $100 \%$ concordant set of tests yields $\mathrm{p}<0.01$ is 3 , confirming that the tested population sample yielded group sizes adequate to support the test of this hypothesis

\section{Discussion}

The synthesis and purification of all compounds was efficient, with yields around $85 \%$. Our initial screening efforts focused on the three compounds in Table 1 with the lowest cLogP values (ET6-17, ET6-47 and ET6-21). ET6-17 and ET6-21 presented spectroscopic properties compatible with our in vitro binding assays and were tested for amyloid fibril 
binding and specificity (Figures $1 \mathrm{~A}$ and B). Regression of the binding constants (Supplemental figure S3) yielded micromolar binding constants, compared to the known nanomolar binding constants of Methoxy-XO4, SB-13, and AV-45, suggesting that amyloid binding ability is significantly affected by the hydrophilicity of these molecules. Interestingly however, compared to similar studies performed with XO4[15,17] these molecules have only about $60 \%$ of the bound species displaced by Chrysamine G, compared to $>80 \%$ for XO4. This suggests that they may be binding a slightly different site than methoxy-XO4. ET6-21 was chosen as the lead candidate based on its lower cLogP value and marginally higher amyloid binding ability.

The hydrophilicity of ET6-21 (and the ampiphilicity of ET3-73) leads to the formation of stable liposomes, without bilayer compromise, as indicated in Table 2. Liposomes with saturated lipid and cholesterol in the bilayer, when extruded through $100 \mathrm{~nm}$ membranes, result in liposomes with diameter around $100 \mathrm{~nm}$ [20]. Particle sizes considerably larger than $100 \mathrm{~nm}$ are associated with bilayer destabilization, vesicle fusion, and the formation of multilamellar structures, as demonstrated with Methoxy-XO4 liposomes in Table 2 and Supplemental Figure S2. The relatively hydrophobic XO4 ligand, when tethered onto a liposome surface by a flexible PEG tether, should have a tendency to partition into the bilayer and therefore compromise bilayer stability. Upon replacement of the Methoxy-XO4 ligand with the more hydrophilic ET6-21 ligand, there is no longer a propensity for the ligand to partition into the bilayer, thus retaining bilayer integrity. This is consistent with the $148.9 \pm 19.5 \mathrm{~nm}$ particle size and low polydispersity $(0.08 \pm 0.05)$ observed with ET3-73 targeted liposomes (Table 2).

To build a nanoparticle with sufficient T1 signal to allow visualization of amyloid plaques, we adopted the Dual-Gd presentation previously demonstrated to induce enhanced Gd relaxivity $[15,17]$. The conjugation of Gd chelates to a bis-stearyl amide anchor inserted into the bilayer, retards rotational correlation of the $\mathrm{Gd}$ atom, thereby increasing the rotational correlation time $\tau_{R}$. It has been shown by Caravan $[16,21,22]$ via solution of the SolomonBlombergen-Morgan equations that increased rotational correlation times of Gd chelates lead to a peak in $\mathrm{T} 1$ relaxivity at low field strengths, around $1 \mathrm{~T}$, while this enhancement is greatly reduced, and eventually is reversed, at higher field strengths $>7 \mathrm{~T}$. This is consistent with the behavior observed (Figure 2A), the T1 relaxivity of liposomal surface Gd (on a Gd molar basis) is $2.5 \mathrm{x}$ higher than that of a free molecular chelate at $1.4 \mathrm{~T}$ and $1.5 \mathrm{~T}$, but at higher fields, the free molecular chelate exhibits a higher relaxivity. We therefore chose to test relaxivity, and perform in vivo imaging at a low (1T) field strength.

When a targeted liposome, such as the ET3-73 targeted liposome, binds to a molecular target such as the beta sheet structure of an amyloid plaque, each molecular target is associated with an entire liposome, and the corresponding signal in the MR image is attributable to the entire liposome. The relaxivity on a liposome molar basis is therefore linearly related to the signal associated with the molecular target. We therefore measured the relaxivity of surface $\mathrm{Gd}$ liposomes on a liposome molar basis, as a function of increasing $\mathrm{Gd}$ chelate concentration in the liposome bilayer. The results in Figure $2 \mathrm{~B}$ show an expected linear dependence of relaxivity on the number of Gd chelates in the bilayer. At $25 \%$ of the bilayer molecules bearing a Gd chelate, the T1 relaxivity on a per-particle basis is estimated 
to be around $180,000 \mathrm{mM}^{-1} \mathrm{~s}^{-1}$. For $\sim 140 \mathrm{~nm}$ diameter particles used here (see Figure Table 2), and assuming a per-lipid cross sectional area of $65 \AA^{2}$, this corresponds to roughly 48,000 Gd chelates per particle, of which half are on the outer leaflet of the liposome bilayer, and half on the inner leaflet. Assuming that the inner leaflet $\mathrm{Gd}$ is not substantially active for $\mathrm{T} 1$ relaxation while the outer leaflet chelates are, this suggests a molar relaxivity on a per-Gd basis of roughly $8 \mathrm{mM}^{-1} \mathrm{~s}^{-1}$, of the same order as that measured using the $1.4 \mathrm{~T}$ relaxometer (Figure 2A).

Upon injection into the bloodstream, the ET3-73 targeted liposomes avidly cross the blood brain barrier and bind to amyloid plaques (Figure 3). This somewhat surprising permeability of the blood brain barrier is however, consistent with our past observations $[15,16]$ where we noted the ability of Methoxy-XO4 targeted liposomes to cross the BBB and label amyloid plaques in the APP/PSEN1 mouse model of AD. In a recent study [16,23], we examined the ability of untargeted liposomes to cross the BBB in the TetO/APP model of AD, and demonstrated significant leakage across the BBB in age-dependent and amyloid-dependent manners. While all mice tested showed leaks in the choroid plexus, older mice (>14 months of age) showed significantly higher leak along major blood vessels, while APP+ mice showed greater leak in the cerebral cortex. The current observations are consistent with previous work [16,24], the TetO/APP mice in the present study were all >14 months old and show more widespread signal in the brain, while the Tg2576 mice were all 9-10 months old and show less signal, concentrated around pial and cortical vessels. Further, while 6/6 TetO/APP mice showed clear signal in the brain, consistent with localization of the targeted agent, only 2/6 Tg2576 animals showed clear brain signal. Immunohistochemically, however, the same $2 / 6 \mathrm{Tg} 2576$ animals exhibited amyloid pathology, while the remaining $4 / 6$ did not, confirming that the MR signal was $100 \%$ concordant with the gold-standard immunohistochemical observations across all 20 animals tested.

Confirmation of the localization of the particles to amyloid plaques comes from the data in Figure 4. Whole brain near-IR images visualizing the Indocyanine label (4C and D) are consistent with increased localization in APP+ brains compared to APP- brains. Slices through the cortex and hippocampus of $\mathrm{APP}+$ and $\mathrm{APP}-$ mice (4A and $\mathrm{B}$ ) show significant localization in the APP+ brain, with the maximum intensity observed in the cerebral cortex and portions of the hippocampus. Significant staining is also observed in the thalamus, and is consistent with thalamic signal in the MR image (Figure 4C) of the same mouse. Under high magnification (Figures 4E and 4F) of the cerebral cortex, the ICG signal in the APP+ mouse brain is seen to be both punctate (i.e. consistent with labeling of focal plaques) and along blood vessel walls (i.e. consistent with labeling of CAA).

Detailed histological examination of individual focal plaques (Figures $4 \mathrm{G}$ and $4 \mathrm{H}$ ) in the cerebral cortex reveal that the ligand targeted particles appear to label both the dense and diffuse sections of the plaque. In these figures, the 4G8 antibody, visualized with FITC is labeled green, the ICG is labeled red, while the ET6-21 ligand is labeled blue. Figure 4G shows a field with 3 focal plaques visible. Also in the field is a more diffuse surrounding amyloid deposition pattern. The 4G8 antibody labels both these entities. The focal plaques appear to have a relatively uniform amyloid density at the center, and higher density in the periphery, consistent with numerous other observations of plaques in mice using antibody 
and thioflavin-S staining, e.g.[23,25] The ICG and ET6-21 labels (red and blue respectively) are highly correlated with each other and with the antibody label, demonstrating a low density of binding in the center of the plaques and high density nodules in the periphery, leaving little doubt that the liposomal particles bind intact, to the plaques, and label both the high density focal plaques as well as lower density diffuse plaques. Although there is substantial overlap between channels, differences are noted in the intensity and distribution of 4G8, ICG, and ET6-21 within plaques, suggesting differences in binding specificity associated with amyloid deposits; 4G8 binds to monomeric, oligomeric, and aggregate forms of A $\beta[26]$, whereas the ET6-21 ligand, similar to other thioflavin-like molecules, preferentially binds to aggregate and fibrillar forms.

We noted that there were some differences in the MRI signal patterns between the TetO/APP and TG2576 mice, with the former showing signal in the cerebral cortex, hippocampus, and striatum, consistent with preferential amyloid deposition in these areas due to heterogeneous activity of the CaMkIIa promoter [15,16,24], while a more complete deposition pattern is observed in the $\mathrm{Tg} 2576$ mice, consistent with the more uniform plaque deposition expected in this model $[16,25]$. The Tg2576 mice also showed strong ventricular signal, most likely due to the continued presence of the agent in the cerebrospinal fluid, the likely transport medium for the extravasated liposomes. As a confirmation that ET6-21 can bind amyloid at levels typically observed in human brains, and in a spontaneous disease model other than the mouse overexpression system, we note that it binds to amyloid pathology in both aged canine brain and in human brain tissue slices, as demonstrated in Figure 1C and 1D.

That liposomes penetrate the BBB in mouse models of amyloid deposition and Alzheimer's disease is no longer in question: our previous work $[15,16]$ and the current work have collectively proven this in 3 different mouse models. (APP/PSEN1, TetO/APP, Tg2576). This penetration does not require any active transport mechanism, our past work [16] tested simple PEGylated liposomes, and demonstrated their avid transport through the BBB. Retention of the particles at the sites of amyloid deposition, for extended periods of time, however, depends on their binding to a molecular target. The use of Methoxy-XO4 as the binding ligand in our previous work demonstrated binding to amyloid plaques and visualization ex vivo by microscopy. In the current work, the use of a novel targeting ligand ET6-21 enabled the preparation of liposomes with improved bilayer integrity, and the consequent encapsulation of large amounts of Gd chelates. These Gd chelates, presented on the surface of the liposome, are hyper-relaxive at low $(\sim 1 \mathrm{~T})$ field strengths, and enable perparticle relaxivity of $\sim 180,000 \mathrm{mM}^{-1} . \mathrm{s}^{-1}$, sufficient to enable their visualization in vivo using T1-weighted MRI. Thus, amyloid plaques in two different mouse models (Tg2576 and TetO/APP) are visualized.

\section{Limitations of the present work}

Many of the mice used in this study were rather old (>15 Months) and had highly variable amounts of fat around the neck, along with numerous skin lesions, around the head and body. Some of the TetO/APP mice also exhibited aggressive behavior, and before being isolated, would fight with littermates, leading to cuts and lacerations. Fat is intrinsically bright in T1 images, and the liposomal contrast agent is expected to localize into sites of 
inflammation, infection, and vascular damage. Additionally, the extracranial amyloid deposits in these animals, including in the vasculature, were not assessed. Therefore, it is possible that bright signal in both the pre and post-contrast images could occur in the extracranial anatomy. Such signal was indeed observed in some of the mice, and constitutes a limitation of this work. Of note however, is that in every single APP+ animal treated with the targeted nanoparticles, the intracranial signal did indeed rise after the treatment, consistent with amyloid labeling.

An important open question is if a similar MRI agent can be developed for use in humans. The human BBB shares some similarities with the mouse BBB, but it is not yet known whether this agent will penetrate the human BBB sufficiently. That said, MRI studies performed in $\mathrm{AD}$ and $\mathrm{MCI}$ patients have also indicated that the $\mathrm{BBB}$ is indeed compromised $[27,28]$ and the extent of compromise is independent of amyloid burden. A recent study confirmed [29], using DCE-MRI that the BBB in the aging human hippocampus breaks down, and becomes permeable. Confirmation that the agent binds amyloid pathology other than in a mouse overexpression model has been demonstrated by testing binding to amyloid deposits in dog brain and human brain slices in vitro. Image resolution also constitutes an open question. In the current work, in-plane resolution was about $150 \mu \mathrm{m}$, finer than typically used in human studies, but technically feasible.

Gadolinium contrast agents have recently been shown to dissociate free Gd from the chelates, and the free Gd then deposit in the brain (see for example [30]), and the FDA is investigating its long term effects [31]. The dissociation and brain deposition is shown to increase when low affinity chelators are used. In this work, we did indeed use such low affinity chelators, and the use of an agent such as ours in humans would not be advisable unless a change to a high affinity macrocyclic chelator is made. A reformulation to change the chelator is currently being conducted, and results using that agent will be reported in future work.

\section{Conclusion}

Our work provides proof-of-concept MRI imaging of amyloid plaques in rodent models at clinically acceptable field strengths, and suggests that development of an MRI agent for clinical diagnosis of amyloidopathy in human patients is possible. An MRI agent offers a number of substantial benefits over current non-invasive imaging technologies, such as PET imaging, including increased availability, reduced cost, and enhanced resolution. As of the date of this writing, three PET agents are approved for the imaging of amyloid plaques, but their availability is limited, and restricted to large academic medical centers. MRI based agents offer worldwide availability; and T1 agents are extremely attractive because of their positive signal, leading to increased confidence in signal interpretation. The agent is optimized for use in low field (1-3T) scanners, atypical for small animal work, but completely consistent with the state-of-the-art for human imaging, boding well for translation to clinical testing.

Diagnostic utility could well come in combination with other tests. While the imaging of amyloid pathology, in isolation, may not be diagnostic for $\mathrm{AD}$, it is probable that when 
combined with a secondary marker, such as tau neurofibrillary tangles, the specificity for AD is likely to be increased, and possibly sufficient for diagnosis of AD. PET agents for tau are under investigation, but even if they are successful, diagnosis of AD with PET agents would require two separate PET scans, with the accompanying availability and geographical distribution challenges for both agents. A MRI based amyloid imaging agent such as the one described in this work, could potentially be a first screen, be globally available, and identify those patients who are candidates for a second, possibly less widely available, tau screen.

\section{Supplementary Material}

Refer to Web version on PubMed Central for supplementary material.

\section{Acknowledgments}

The authors thank Dr. Michael Weiner (University of California, San Francisco) for numerous discussions of the results of this study. Portions of this study were supported by NIA of the National Institutes of Health under award number 1R15AG039008 (J.L.E). Human tissue was obtained from the The UK-ADC (P30AG28383) and canine tissue was acquired under R01AG031764 to EH.

\section{Bibliography}

1. Joshi AD, Pontecorvo MJ, Adler L, Stain MG, Skovronsky DM, Carpenter AP, Mintun MA, Florbetapir F. 18 study investigators. Radiation dosimetry of florbetapir F 18. EJNMMI Res. 2014; 4:4. [PubMed: 24401181]

2. Huda W, Tipnis SV. DOSES METRICS AND PATIENT AGE IN CT. Radiation protection dosimetry. 2015:1-7.

3. [Accessed July-December 2015] usa.healthcare.siemens.com

4. Agyare EK, Jaruszewski KM, Curran GL, Rosenberg JT, Grant SC, Lowe VJ, Ramakrishnan S, Paravastu AK, Poduslo JF, Kandimalla KK. Engineering theranostic nanovehicles capable of targeting cerebrovascular amyloid deposits. J Control Release. 2014; 185:121-129. [PubMed: 24735640]

5. Wadghiri YZ, Li J, Wang J, Hoang DM, Sun Y, Xu H, Tsui W, Li Y, Boutajangout A, Wang A, de Leon M, Wisniewski T. Detection of Amyloid Plaques Targeted by Bifunctional USPIO in Alzheimer's Disease Transgenic Mice Using Magnetic Resonance Microimaging. PLoS ONE. 2013; 8:e57097. [PubMed: 23468919]

6. Koffie RM, Farrar CT, Saidi L-J, William CM, Hyman BT, Spires-Jones TL. Nanoparticles enhance brain delivery of blood-brain barrier-impermeable probes for in vivo optical and magnetic resonance imaging. Proc Natl Acad Sci USA. 2011; 108:18837-18842. [PubMed: 22065785]

7. Yang J, Wadghiri YZ, Hoang DM, Tsui W, Sun Y, Chung E, Li Y, Wang A, de Leon M, Wisniewski T. Detection of amyloid plaques targeted by USPIO-A $\beta 1-42$ in Alzheimer's disease transgenic mice using magnetic resonance microimaging. Neuroimage. 2011; 55:1600-1609. [PubMed: 21255656]

8. Higuchi M, Iwata N, Matsuba Y, Sato K, Sasamoto K, Saido TC. 19F and 1H MRI detection of amyloid beta plaques in vivo. Nat Neurosci. 2005; 8:527-533. [PubMed: 15768036]

9. Viola KL, Sbarboro J, Sureka R, De M, Bicca MA, Wang J, Vasavada S, Satpathy S, Wu S, Joshi H, Velasco PT, MacRenaris K, Waters EA, Lu C, Phan J, Lacor P, Prasad P, Dravid VP, Klein WL. Towards non-invasive diagnostic imaging of early-stage Alzheimer's disease. Nat Nanotechnol. 2015; 10:91-98. [PubMed: 25531084]

10. Kim J-H, Ha TL, Im GH, Yang J, Seo SW, Lee IS, Lee JH. Magnetic resonance imaging of amyloid plaques using hollow manganese oxide nanoparticles conjugated with antibody a $\beta 1-40$ in a transgenic mouse model. Neuroreport. 2013; 24:16-21. [PubMed: 23168564]

11. Kim J-H, Ha TL, Im GH, Yang J, Seo SW, Chung JJ, Chae SY, Lee IS, Lee JH. Magnetic resonance imaging for monitoring therapeutic response in a transgenic mouse model of 
Alzheimer's disease using voxel-based analysis of amyloid plaques. Neuroreport. 2014; 25:211218. [PubMed: 24518227]

12. Amatsubo T, Morikawa S, Inubushi T, Urushitani M, Taguchi H, Shirai N, Hirao K, Kato M, Morino K, Kimura H, Nakano I, Yoshida C, Okada T, Sano M, Tooyama I. Trifluoromethoxybenzylated ligands improve amyloid detection in the brain using (19)F magnetic resonance imaging. Neurosci Res. 2009; 63:76-81. [PubMed: 18996420]

13. Nabuurs RJA, Kapoerchan VV, Metaxas A, de Jongh S, de Backer M, Welling MM, Jiskoot W, Windhorst AD, Overkleeft HS, van Buchem MA, Overhand M, van der Weerd L. Polyfluorinated bis-styrylbenzenes as amyloid- $\beta$ plaque binding ligands. Bioorg Med Chem. 2014; 22:2469-2481. [PubMed: 24657049]

14. Tooyama, I.; Yanagisawa, D.; Taguchi, H.; Kato, T.; Hirao, K.; Shirai, N.; Sogabe, T.; Ibrahim, NF.; Inubushi, T.; Morikawa, S. Amyloid imaging using fluorine-19 magnetic resonance imaging (19FMRI). Ageing Research Reviews. 2016. http://dx.doi.org/10.1016/j.arr.2015.12.008, preprint ahead of publication

15. Tanifum EA, Dasgupta I, Srivastava M, Bhavane RC, Sun L, Berridge J, Pourgarzham H, Kamath R, Espinosa G, Cook SC, Eriksen JL, Annapragada A. Intravenous delivery of targeted liposomes to amyloid- $\beta$ pathology in APP/PSEN1 transgenic mice. PLoS ONE. 2012; 7:e48515. [PubMed: 23119043]

16. Tanifum EA, Starosolski ZA, Fowler SW, Jankowsky JL, Annapragada AV. Cerebral vascular leak in a mouse model of amyloid neuropathology. J Cereb Blood Flow Metab. 2014:1-9. [PubMed: 25352045]

17. Ghaghada KB, Ravoori M, Sabapathy D, Bankson J, Kundra V, Annapragada A. New dual mode gadolinium nanoparticle contrast agent for magnetic resonance imaging. PLoS ONE. 2009; 4:e7628. [PubMed: 19893616]

18. Cotman CW, Head E. The canine (dog) model of human aging and disease: dietary, environmental and immunotherapy approaches. J Alzheimers Dis. 2008; 15:685-707. [PubMed: 19096165]

19. Barenholz Y. Doxil ${ }^{\circledR}-$ the first FDA-approved nano-drug: lessons learned. J Control Release. 2012; 160:117-134. [PubMed: 22484195]

20. Sood, S. Thesis. San Jose State University; San Jose, CA: 1999. Characterization of Liposome Manufacturing Using Extrusion.

21. Caravan P, Ellison JJ, McMurry TJ, Lauffer RB. Gadolinium(III) Chelates as MRI Contrast Agents: Structure, Dynamics, and Applications. Chem Rev. 1999; 99:2293-2352. [PubMed: 11749483]

22. Caravan P, Farrar CT, Frullano L, Uppal R. Influence of molecular parameters and increasing magnetic field strength on relaxivity of gadolinium- and manganese-based $\mathrm{T} 1$ contrast agents. 2009; 4:89-100.

23. Kuo YM. Comparative Analysis of Amyloid-beta Chemical Structure and Amyloid Plaque Morphology of Transgenic Mouse and Alzheimer's Disease Brains. Journal of Biological Chemistry. 2001; 276:12991-12998. [PubMed: 11152675]

24. Jankowsky JL, Slunt HH, Gonzales V, Savonenko AV, Wen JC, Jenkins NA, Copeland NG, Younkin LH, Lester HA, Younkin SG, Borchelt DR. Persistent Amyloidosis following Suppression of A $\beta$ Production in a Transgenic Model of Alzheimer Disease. 2005; 2:e355.

25. Hosono T, Nishitsuji K, Nakamura T, Jung C-G, Kontani M, Tokuda H, Kawashima H, Kiso Y, Suzuki T, Michikawa M. Arachidonic acid diet attenuates brain A $\beta$ deposition in $\mathrm{Tg} 2576$ mice. Brain Res. 2015; 1613:92-99. [PubMed: 25881896]

26. Kim KS, Miller DL, Sapienza VJ, Chen C, Bai C. Production and characterization of monoclonal antibodies reactive to synthetic cerebrovascular amyloid peptide. Neuroscience research communications. 1988; 2:121-130.

27. Wang H, Golob EJ, Su M-Y. Vascular volume and blood-brain barrier permeability measured by dynamic contrast enhanced MRI in hippocampus and cerebellum of patients with MCI and normal controls. Journal of magnetic resonance imaging : JMRI. 2006; 24:695-700. [PubMed: 16878309]

28. Starr JM, Farrall AJ, Armitage P, McGurn B, Wardlaw J. Blood-brain barrier permeability in Alzheimer's disease: a case-control MRI study. Psychiatry Res. 2009; 171:232-241. [PubMed: 19211227] 
29. Montagne A, Barnes SR, Sweeney MD, Halliday MR, Sagare AP, Zhao Z, Toga AW, Jacobs RE, Liu CY, Amezcua L, Harrington MG, Chui HC, Law M, Zlokovic BV. Blood-brain barrier breakdown in the aging human hippocampus. Neuron. 2015; 85:296-302. [PubMed: 25611508]

30. Montagne A, Toga AW, Zlokovic BV. Blood-Brain Barrier Permeability and Gadolinium: Benefits and Potential Pitfalls in Research. JAMA Neurol. 2016; 73:13-14. [PubMed: 26524294]

31. Center for Drug Evaluation. [accessed December 2015] Research Drug Safety and Availability FDA Drug Safety Communication: FDA evaluating the risk of brain deposits with repeated use of gadolinium-based contrast agents for magnetic resonance imaging (MRI). fda.gov 

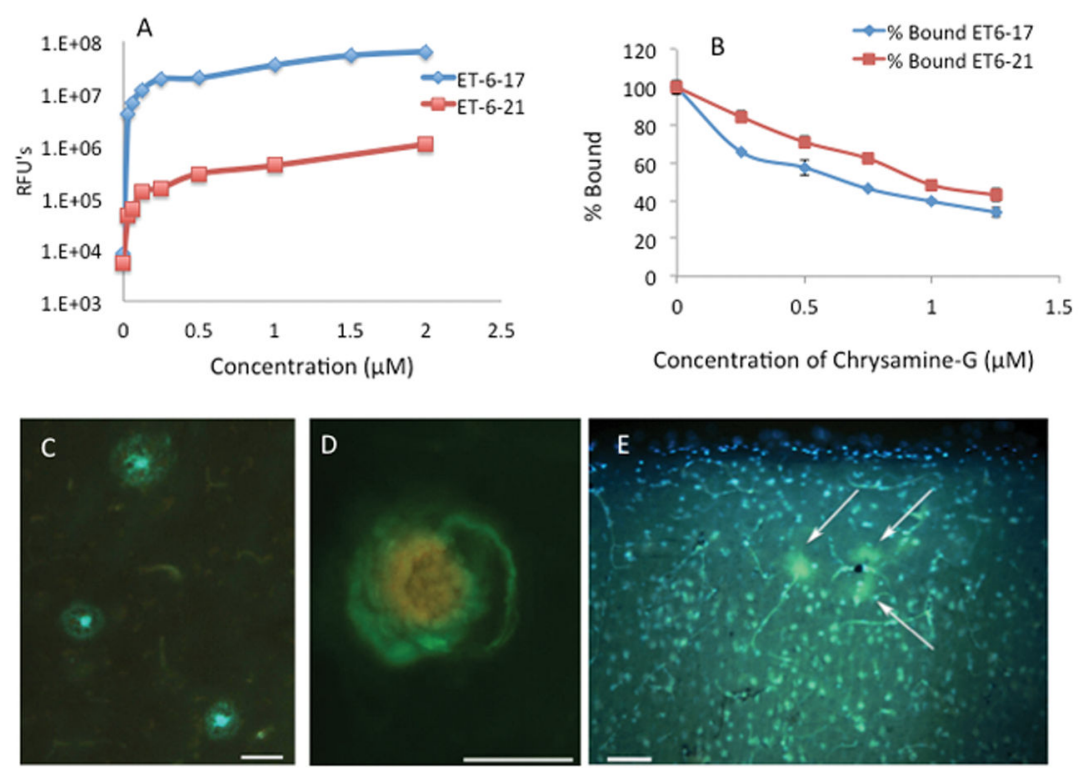

Figure 1.

(A) Novel hydrophilic amyloid binding compounds ET6-17 and ET6-21 (LogP 1.80 and 1.06 respectively) bind to amyloid fibrils. $\mathrm{N}=3$ at each point. (B) A competitive binding study holding the bulk phase concentration constant at $1.5 \mu \mathrm{M}$ shows that binding is competitive with Chrysamine-G, consistent with specificity to the Thioflavin binding site. $\mathrm{N}=3$ at each point. (C) ET6-21 specifically stains amyloid plaques (green/blue) in a section from the frontal cortex of an AD autopsy case (D) ET6-21 labels cerebral amyloid angiopathy (green) in the prefrontal aged dog brain (red/yellow labeling is due to blood cells inside the lumen of the blood vessel showing autofluorescence). Images were collected on an Olympus BX-51 epifluorescent microscope using the broadpass filter. (Scale bars represent $50 \mu \mathrm{m}$ length). RFU: Relative Fluorescence Unit. 

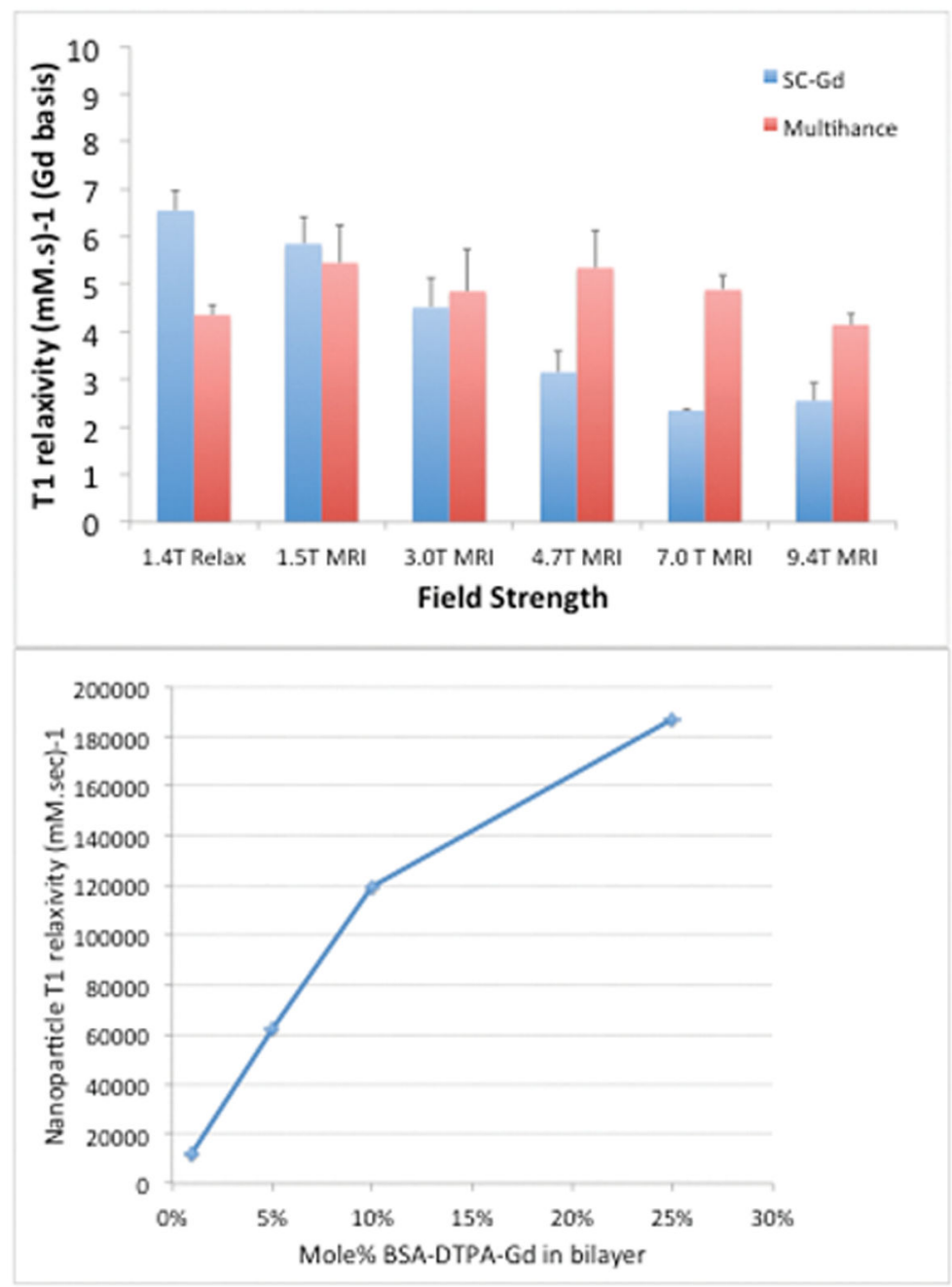

Figure 2.

(A) Surface Gadolinium chelate BisStearoyl Amine-DTPA-Gd on nanoparticles exhibits enhanced relaxivity compared to a free Gd chelate(GadobenateDimelglumine, Multihance ${ }^{\mathrm{TM}}$ ) at lower field strengths, but the difference erodes at higher field strengths to the point where at $9.4 \mathrm{~T}$, the free chelate is more relaxive than the surface presentation. (B) On a particle basis, at $1 \mathrm{~T}$ a relaxivity of $180,000 \mathrm{mmol}^{-1} \mathrm{~s}^{-1}$ is achieved using $25 \mathrm{~mole} \%$ of the BSADTPA-Gd construct. 1.5, 3, 4.7, 7 and 9.4T measurements were made on individual MRI instruments. 1.4T measurement was made on a Bruker minispec mq60 relaxometer. 1T measurements were made on an Aspect M2 permanent magnet MRI instrument. 

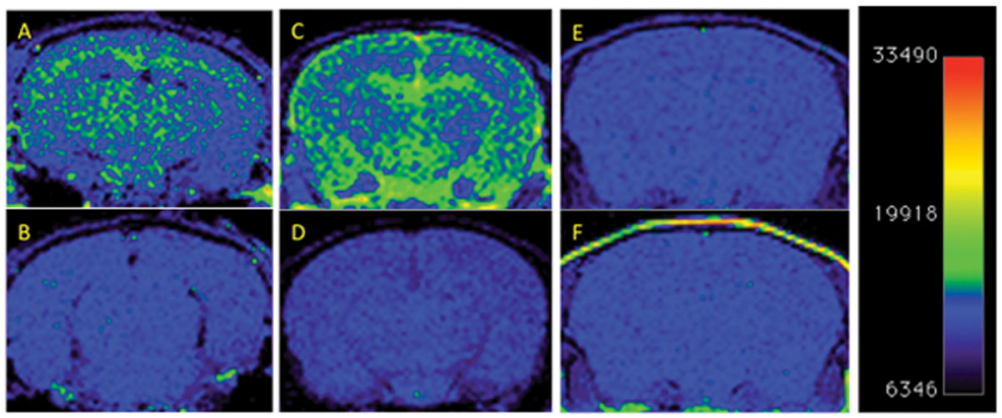

\section{Figure 3.}

T1 weighted MR Imaging of amyloid deposits in mouse models, pre- and 4 days post i.v. injection of nanoparticle MR agent. A 2D Spin-echo sequence, (TE $=32 \mathrm{~ms}, \mathrm{TR}=770 \mathrm{~ms}$, Slice Thickness $=1.2 \mathrm{~mm}, \mathrm{FOV}=30 \times 30 \mathrm{~mm}$, matrix $=156 \times 156, \mathrm{NEX}=2$ ) was used. Coronal $2 \mathrm{D}$ slices of the brain through the cortex and hippocampus are shown. Window Level and Window Width are set identically for each pair of images A-B, C-D, E-F. (A) TetO/APPsweind mouse, 15 months old, APP production suppressed until 6 weeks of age by doxycycline in diet. (B) Pre-injection scan of same mouse. (C) Tg2576 mouse (APPswe) 9 months old, and (D) Pre-injection scan of same mouse. (E) Tg2576 mouse injected with untargeted (non amyloid-binding) particles (F) Non transgenic sibling of E, injected with amyloid-targeted particles. Total signal was mapped to a "rainbow" color map using Osirix software, the range from the predominant blue background to green foreground represents a $>2 \mathrm{x}$ signal increase, and the range from green to yellow a further 2x increase. 9 month old $\mathrm{Tg} 2576$ mice show a different pattern of labeling (pial, hippocampal and some signal in the ventricles) compared to older TetO/APP mice, (extensive cortical and hippocampal signal with no ventricular signal) suggesting slow clearance from the CSF in the Tg2576 example. Images are consistent with 6/6 animals in TetO/APPswe-ind group and 2/6 in the Tg2576 APP+ group. Subsequent immunohistochemical measurements showed that only these $2 / 6 \mathrm{Tg} 2576$ animals had amyloid pathology, while the other $4 / 6$ did not, consistent with the MRI signal. 


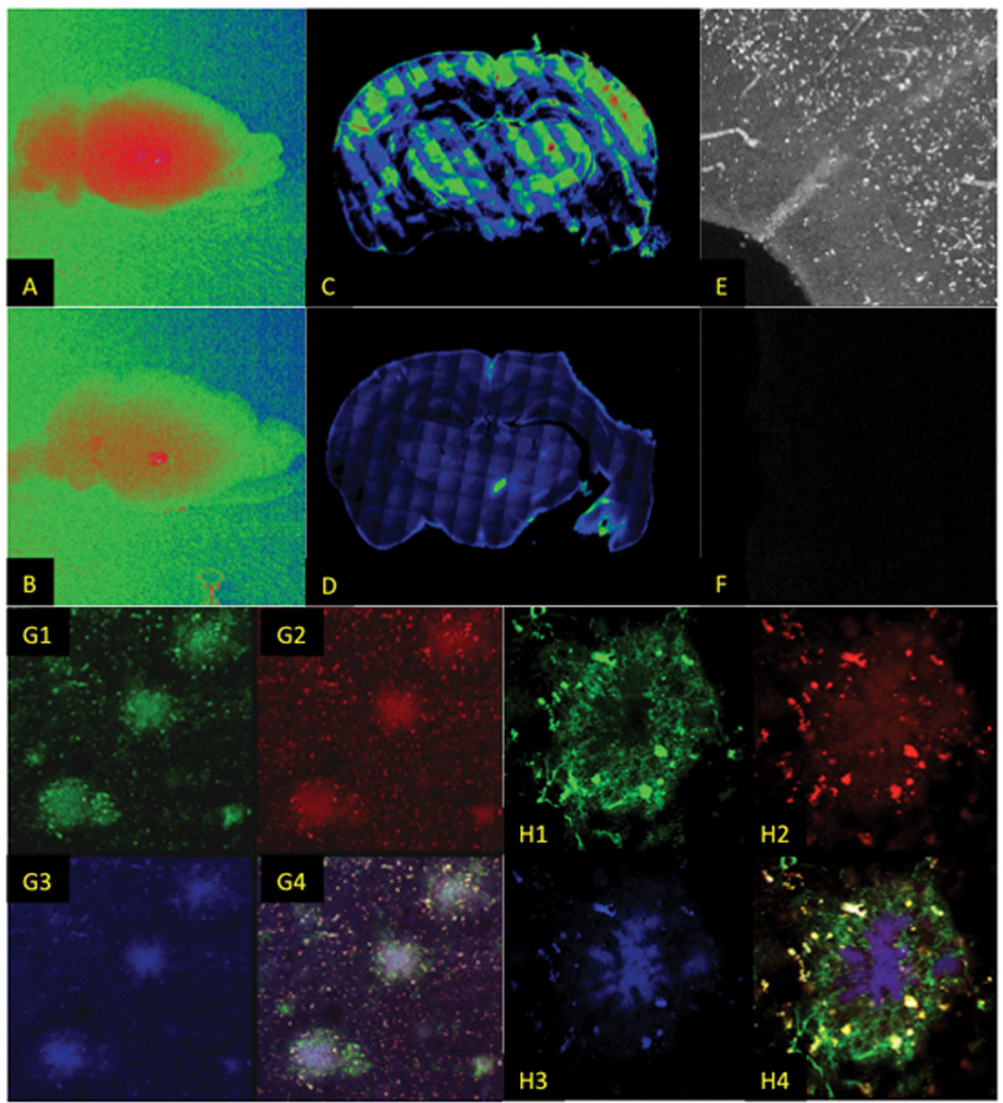

Figure 4.

Fluorescence imaging of brain tissue from mice injected i.v. with amyloid targeted nanoparticles confirms MRI findings. The particles carried 0.5\% Indocyanine Green (ICG) in the bilayer for near-infrared imaging. Whole brains of an APP-positive (A) and APPnegative (B) mouse show uniformly increased ICG signal (Red) localized in the APPpositive brain, compared to overall lower signal in the APP-negative brain with occasional hotspots possibly corresponding to vascular leak locations. Montaged confocal images of Tg2576 (C) and APP- (D) brains showing greater localization (green, yellow, red) in the APP+ brain. (E) Histological section visualizing ICG showing increased localization in APP positive brain (white) compared to a negative brain $(\mathbf{F})$, showing the punctate structure of the ICG deposit, consistent with localization to plaques. Plaques are stained by the 4G8 antibody (G1, green) and are co-located with the ICG $(\mathbf{G 2}$, red), and the ET6-21 ligand (G3, blue) as shown by the overlay in G4 (10X magnification). At higher magnification (60X) (H1-H4), the co-localization in a single plaque is demonstrated. Of the 17 animals that received the targeted liposomes, $8 / 8$ animals with amyloid pathology as determined by staining with the 4G8 antibody also had positive MR signal, and positive ICG signal, while 9/9 animals with no amyloid pathology as determined by 4G8 staining also had no MR signal or ICG signal. The 3 animals that received the untargeted liposomes all had negative amyloid pathology and no MR or ICG signal. 
Table 1

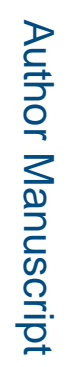

Alternate compounds considered as amyloid plaque binders.

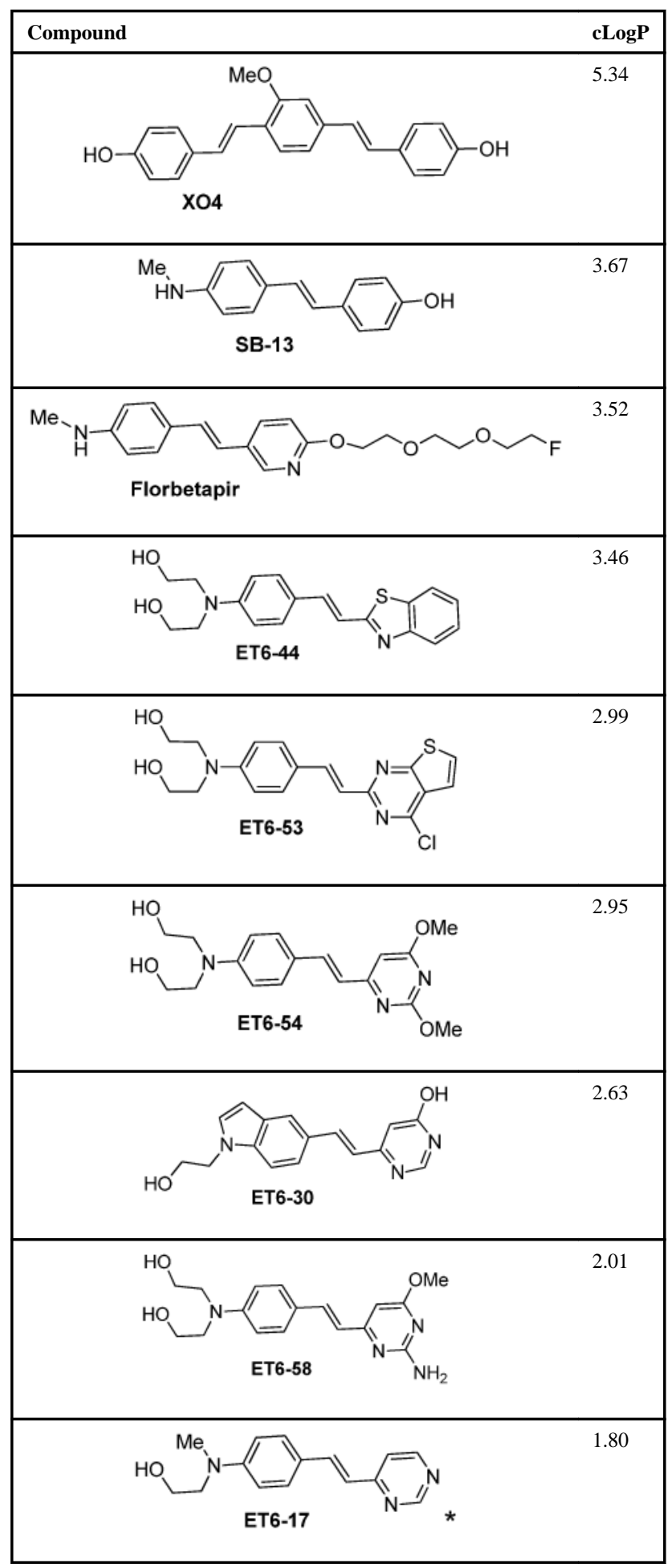

J Alzheimers Dis. Author manuscript; available in PMC 2016 July 04. 


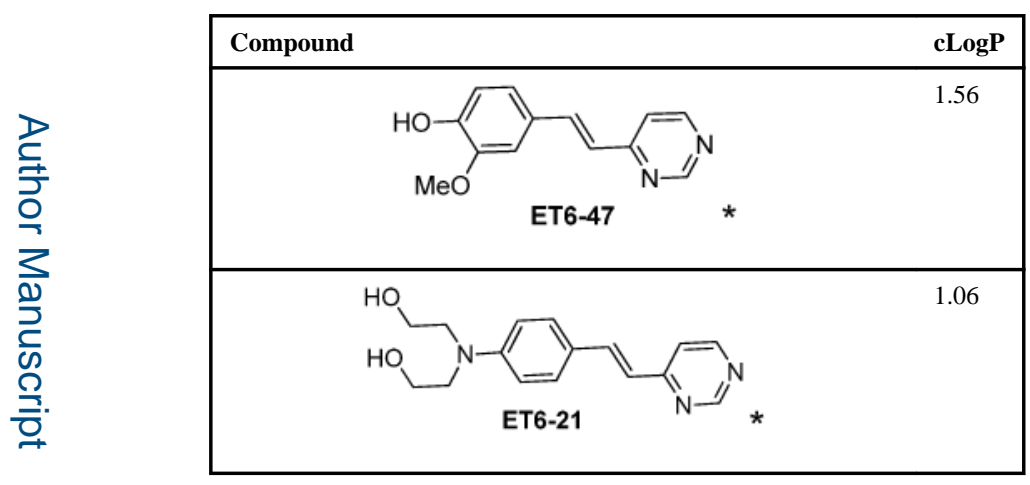

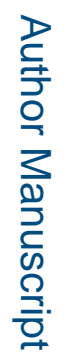

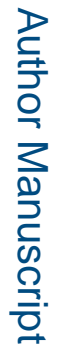

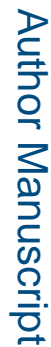




\section{Table 2}

Diameter and polydispersity of liposomes prepared with different surface ligands, after 5 extrusion passes through 400/200 nm filters and 5 extrusion passes through $100 \mathrm{~nm}$ filters. Hydrophobic ligands like XO4 reenter the bilayer and compromise its integrity, as reflected in the larger diameter of the resulting particles. A more hydrophilic ligand like ET6-21 maintains bilayer integrity, and particle size post-extrusion. Data for three preparations of each formulation are presented. BSA: Bis stearoyl amine.

\begin{tabular}{|lcc|}
\hline Bilayer Formulation & Mean Diameter $(\mathbf{n m})$ & Polydispersity Index \\
\hline DSPE-PEG, BSA-DTPA-Gd, DSPE- EG-XO4 & $223.5 \pm 12.6$ & 0.08 \\
\hline DSPE-PEG, BSA-DTPA-Gd & $131.9 \pm 5.8$ & $0.13 \pm .05$ \\
\hline DSPE-PEG, BSA-DTPA-Gd, DSPE-PEG-ET6-21 & $148.9 \pm 19.5$ & $0.08 \pm .05$ \\
\hline
\end{tabular}




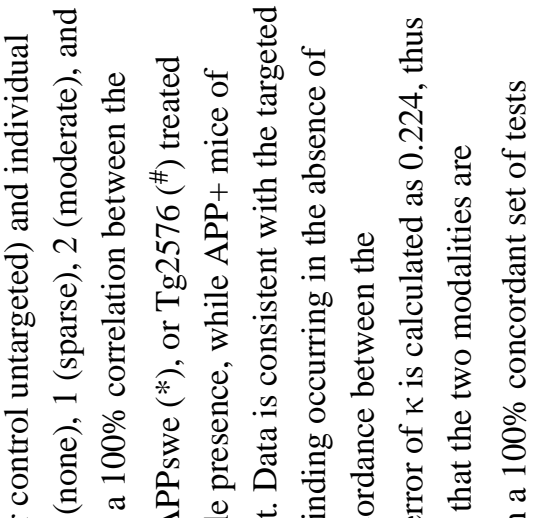

t)

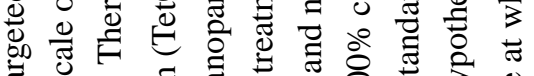

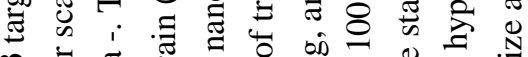

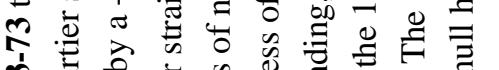

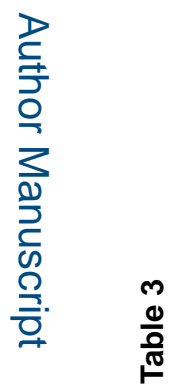

¿

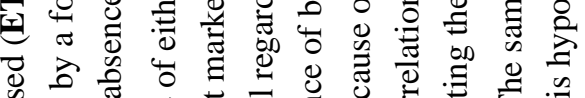

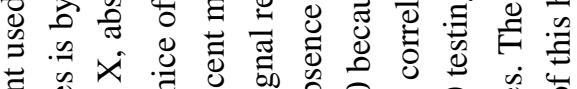

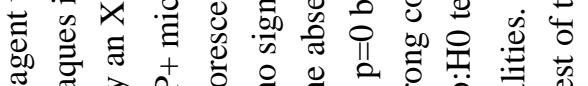

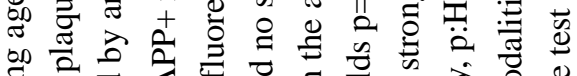

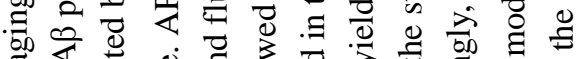

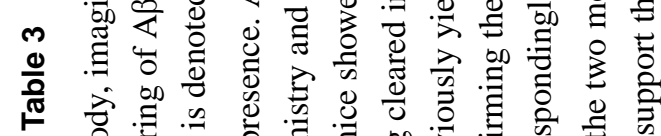

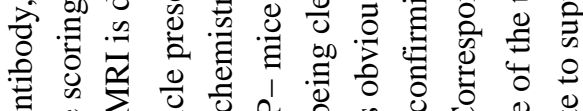

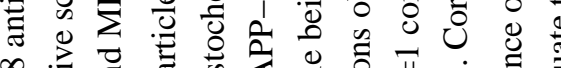

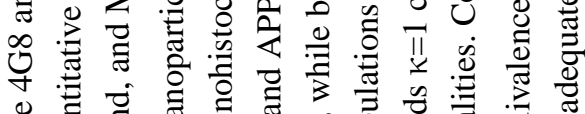

ב

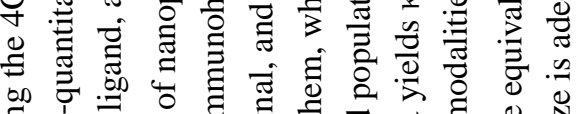

粷

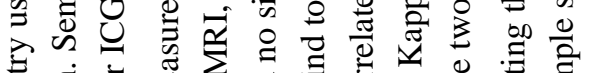

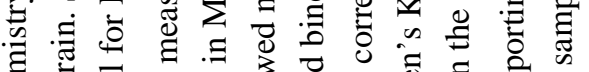

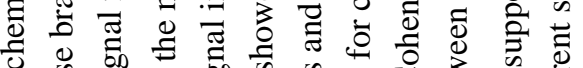

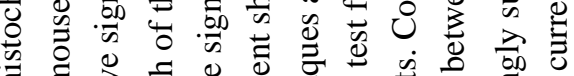

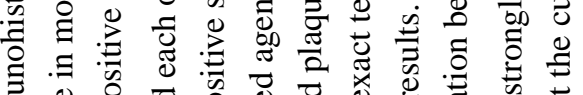

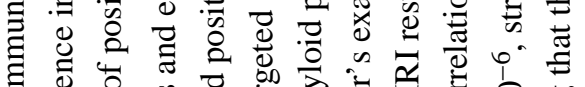

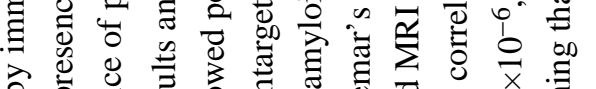

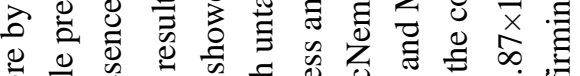

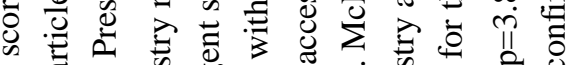
ठิ 을 छ ए

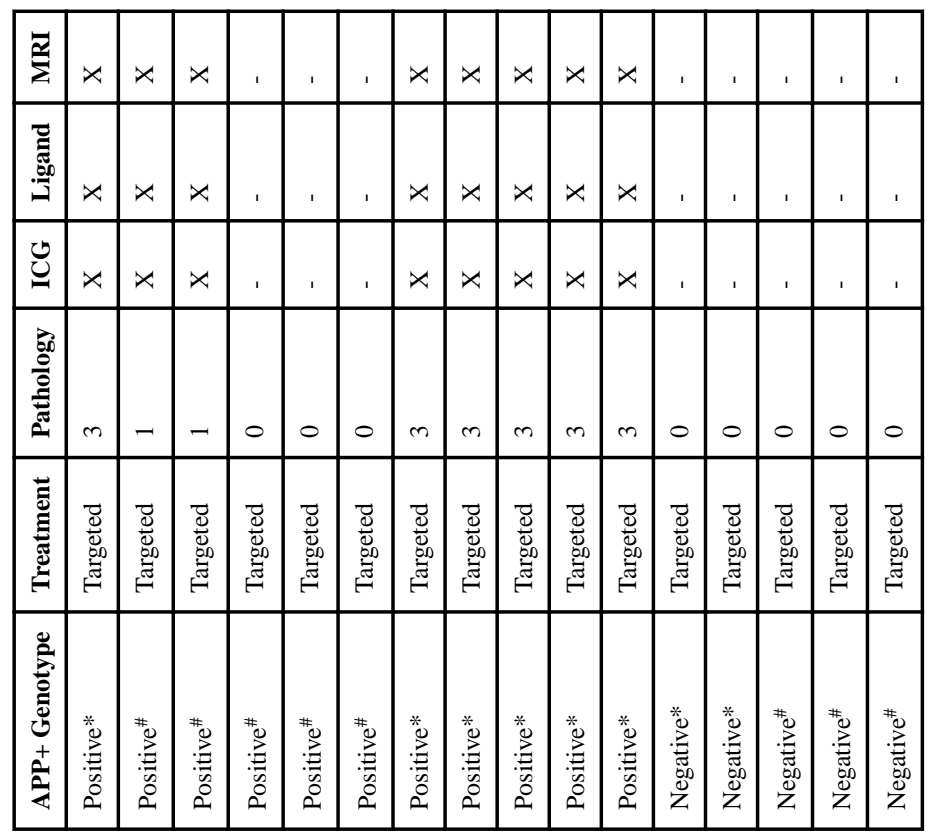




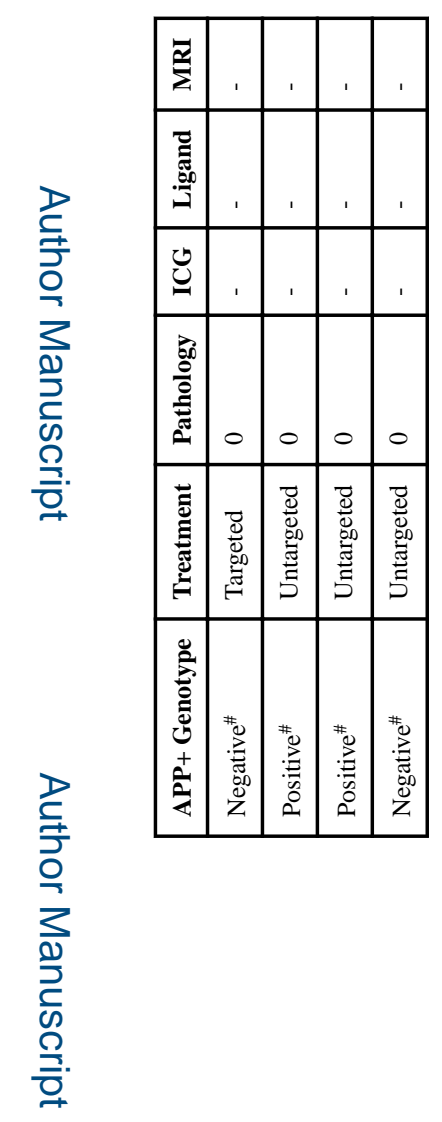

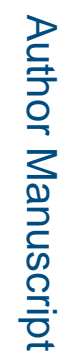

로을 